\title{
Measuring Training Load in Dance: The Construct Validity of Session-RPE
}

\author{
Brenton Surgenor, MSc, ${ }^{1}$ and Matthew Wyon, PhD ${ }^{2,3}$
}

OBJECTIVE: The session rating of perceived exertion (session-RPE) is a practical and non-invasive method that allows a quantification of internal training load (ITL) in individual and team sports. As yet, no study has investigated its construct validity in dance. This study examines the convergent validity between the session-RPE method and an objective heart rate (HR)-based method of quantifying the similar ITL in vocational dance students during professional dance training. METHODS: Ten dance students (4 male, 20 \pm 1.16 yrs; 6 female, $20 \pm 0.52$ yrs) participated in this study. During a normal week of training, session-RPE and HR data were recorded in 96 individual sessions. HR data were analysed using Edwards-TL method. Correlation analysis was used to evaluate the convergent validity between the session-RPE and Edwards-TL methods for assessing ITL in a variety of training modes (contemporary, ballet, and rehearsal). RESULTS: The overall correlation between individual session-RPE and Edwards-TL was $r=0.72, p<0.0001$, suggesting there was a statistically significantly strong positive relationship between session-RPE and Edwards-TL. This trend was observed across all the training modes: rehearsal sessions $(r=0.74, p=0.001)$, contemporary ( $r=0.60, p=0.001)$, and ballet $(r=0.46, p=0.018)$ sessions. CONCLUSIONS: This study shows that session-RPE can be considered as a valid method to assess ITL for vocational dance students, and that notably there is some variation between session-RPE and HR-based TL in different dance activities. Med Prob/ Perform Art 2019;34(1):1-5.

$\mathrm{T}$ o optimize performance in dance, there needs to be a balance between training stimuli and recovery. ${ }^{1,2}$ However, within the context of vocational dance train-

\begin{abstract}
From the ${ }^{1}$ School of Dance, Hong Kong Academy of the Performing Arts, Hong Kong; ${ }^{2}$ Research Centre for Sport, Exercise and Performance, Institute of Sport and Health Sciences, University of Wolverhampton, Wolverhampton, UK; and 3 National Institute of Dance Medicine and Science, UK.
\end{abstract}

The authors declare no funding or conflicts of interest related to this study.

Address for correspondence: Prof. Matthew Wyon, Research Centre for Sport, Exercise and Performance, Institute of Sport and Health Sciences, University of Wolverhampton, Gorway Rd., Walsall WS1 3BD, UK. Tel +44-1902-323144.m.wyon@wlv.ac.uk.<Pls confirm mailing address, tel>

https://doi.org/10.21091/mppa.2019.1002

(c) 2019 Science \& Medicine. Www.sciandmed.com/mppa ing, the question arises, how much training is too much, or too little, to optimize performance? Overloading student dancers with high volumes of intense training has the potential to expose them to fatigue and overtrainingrelated injuries. ${ }^{3,4}$ The reverse is also true, as without sufficient overload on the system, no adaptation will occur and no fitness gains will be made. ${ }^{5,6}$ For this reason, establishing an effective way to record and monitor training load is important when balancing improvement of performance with avoidance of injury, overtraining, and burnout. ${ }^{4}$

Applying a periodization model to vocational dance training has been proposed as a means to help prevent overtraining and its link to injury, whilst optimizing the dancer's performance. ${ }^{4}$ This systematic approach to dance training involves progressive cycling of various aspects of a training program with the goal of enhancing performance outcomes ${ }^{5}$ and is, at heart, a quantitative method. This presents the challenge of finding ways to effectively quantitate training load using a single term. ${ }^{\text {? }}$

Monitoring the training load (TL) is fundamental to studying the effects of specific periodization strategies in vocational dance training as well as monitoring the effect of training load on individual dancers ${ }^{4}$. Given the complexity of the training mode interaction, ${ }^{8}$ there is clearly a need for an accurate means of measuring TL. Without an accurate and reliable means of monitoring TL, there is a risk that unsuitable training will be prescribed, which could result in illness (psychological and physiological), physical injuries, and overtraining. ${ }^{1,9}$

Training load can be measured both externally and internally; external training load (ETL) in dance might include the number of hours of training per week or the number of classes, rehearsal, or performances per week. However, ETL does not provide sufficient information on the physiological/psychological response to the ETL as experienced by the individual dancer. Internal training load (ITL) represents the amount of physiological / psychological stress experienced by each individual dancer. The assessment of ITL requires quantification of the intensity of the training stress imposed on the dancer and its duration. Although the duration of a training session is easily measurable in minutes, its intensity can be determined with different methods, such as heart rate (HR) and rate of perceived exertion (RPE). 
HR has been used by a number of researchers to quantify ITL in sport and physical activities. ${ }^{10-13}$ HR demonstrates an almost linear relationship to maximal oxygen consumption $\left(\mathrm{VO}_{2 \max }\right)$ over a wide range of steady-state submaximal exercise intensities ${ }^{14}$ and provides a suitable methodology for quantifying training intensity during most exercise contexts. One of the most commonly used HR-based methods of quantifying training load was developed by Edwards. ${ }^{15}$ Edwards' method for the calculation of TL (Edwards-TL) uses time spent in five pre-defined zones multiplied by corresponding coefficients. This method is the default setting on a Polar HR monitor system and therefore has gained popularity amongst those seeking a convenient means to objectively quantify training load. Edwards-TL has been used to provide evidence of construct validity by a number of studies. ${ }^{12,16-18}$

Using Edwards-TL as a measure of intensity in dance presents a number of challenges. HR transmitter belts are not widely available within vocational dance schools. In addition, HR response can present problems when evaluating intensity during intermittent physical activity that utilizes both the aerobic and anaerobic energy systems. ${ }^{19,20}$

Thus, although HR-based methods provide objective measures of ITL, they may not be practical for dance. An alternative to HR-based methods is session rate of perceived exertion (session-RPE) developed by Foster and colleagues. ${ }^{12}$ Session-RPE requires dancers/athletes to rate the intensity of an entire session subjectively using the RPE category ratio scale (CR-10) developed by Borg, ${ }^{21}$ multiplied by duration (minutes) of the session. This product represents as a single number the magnitude of ITL in arbitrary units (AU).

A number of studies support the validity of session-RPE as an indicator of ITL. Indeed, the construct validity of session-RPE as a means to record ITL has been investigated in several sports and physical activities and was found to correlate well with HR-based methods of quantifying ITL. ${ }^{12,16,17}$

Session-RPE has the potential to benefit a large proportion of the global dance community since it is inexpensive, easy to use, and proven has reliable as a measure of ITL in a variety of training and performance environments. However, to date, no study has examined the construct validity of the session-RPE method for quantifying ITL in dance, where the operational definition of the construct is the ITL. For this reason, the aim of the present study was to evaluate the convergent validity between the session-RPE and an objective HR-based method for assessing the ITL in a variety of training modes with vocational dance students.

\section{METHODS}

\section{Participants}

Ten dance students ( 4 males: $20 \pm 1.16$ yrs, $59.77 \pm 2.9 \mathrm{~kg}$, $168.3 \pm 5.38 \mathrm{~cm}$; 6 females: $20 \pm 0.52 \mathrm{yrs}, 51.3 \pm 1.03 \mathrm{~kg}$, $159.3 \pm 3.39 \mathrm{~cm}$ ) from a contemporary dance vocational program, volunteered to participate in this study. The dancers were in their first year of full-time study. The study was fully approved by the clinical Research Ethics Committee of the University of Wolverhampton. Before the study, dancers received and signed informed consent prior to data collection. All dancers were fully accustomed to the procedures used in this research and were informed they could withdraw from the study at any time without penalty. $<$ AU: give IRB case \#>

\section{Procedures}

The data collection took place during a typical week (5 days) of training (11 dance sessions: 5 contemporary classes, 3 ballet classes, and 3 rehearsals). During each of the different modes of dance training, session-RPE and HR were recorded and analyzed (96 individual sessions). 14 sessions were not recorded due to participant absence.

Monitoring Training Loads-Session-RPE was calculated using Foster's procedure, ${ }^{12}$ which involves multiplying the training duration in minutes by the mean perceived training intensity. The session-RPE scale is based on the Borg Category Ratio (CR-10) RPE scale 22 modified to translate an athlete's perception of effort into a numerical score between 0 and 10 . This test is designed to ask the athlete to respond to a simple question, "How was your workout?," with the goal of getting an uncomplicated response that reflects the athlete's global impression of the physical session. In the present study, the question "how was your workout?" was changed to "how was your class / rehearsal?" to better reflect the dance environment. All dancers had been familiarized to this scale before the start of the study ( 2 weeks preceding the data collection period) and followed standardized instructions for session-RPE that included session-RPE being collected approximately 15 minutes after each class or rehearsal to ensure that the perceived exertion referred to the whole session rather than the most recent (end of session intensity).

Criterion Methods for Quantifying Physical Training Loads - The HR-based method proposed by Edwards ${ }^{15}$ determines internal load by measuring the product of accumulated training duration (minutes) in five HR zones calculated as a percentage of the individual's age-related HR maximum $\left(\mathrm{HR}_{\max }\right)(220-$ age $)$ using a coefficient relative to each zone $\left(50-60 \% \mathrm{HR}_{\max }=1,60-70 \% \mathrm{HR}_{\max }=2,70-80 \% \mathrm{HR}_{\max }\right.$ $=3,80-90 \% \mathrm{HR}_{\max }=4,90-100 \% \mathrm{HR}_{\max }=5$ ) and then summing the results.

Edwards-TL was derived theoretically and not through experimentation, raising the question of the legitimacy of validating the session-RPE method against this HR-based method. However, criterion-related validity of EdwardsTL has been validated against other measures of ITL and found to demonstrate highly significant correlations. In a review article by Borresen and Lambert, ${ }^{23}$ Edwards-TL was listed as an index of training stress alongside Banister's TRIMP. ${ }^{24}$ Indeed, the majority of research analyzing the ecological validity of session-RPE has used either Edwards- 
TABLE 1. Descriptive Statistics of Session-RPE and Edwards TL

\begin{tabular}{|c|c|c|c|c|}
\hline Internal TL & $n$ & Mean \pm SD & Median & (Min, Max) \\
\hline Overall & 96 & $371.00 \pm 179.34$ & 337.56 & $(48.08,723.24)$ \\
\hline Contemporary & 44 & $488.67 \pm 152.95$ & 504.56 & $(150.22,723.24)$ \\
\hline Rehearsal & 26 & $229.01 \pm 165.96$ & 169.08 & $(48.08,6 \mid 3.32)$ \\
\hline \multicolumn{5}{|l|}{ Edwards-TL } \\
\hline Contemporary & 44 & $182.25 \pm 72.52$ & 164.82 & $(57.54,348.9)$ \\
\hline Rehearsal & 26 & $87.69 \pm 57.20$ & 64.1 & $(17.97,250.97)$ \\
\hline
\end{tabular}

TL or Banister's TRIMP. ${ }^{25-27}$ It should be noted that despite the relative simplicity of Banister's TRIMP, it does not distinguish between different levels of training, therefore making it less compatible with the interval nature of dance training. ${ }^{23}$

HR Measurements and Calculations-Training intensity during each dance session was recorded using Polar Team System HR monitors (Polar, Kempele, Finland), with HR recorded every $5 \mathrm{sec}$. After each training session, HR data were downloaded to a computer using Polar Advantage Software (Polar Electro, Oy, Finland).

\section{Data Analysis}

Descriptive statistics of session-RPE and Edwards-TL were computed and presented; normality of the data was confirmed via quantile-quantile plots.

The following data analyses were conducted for sessionRPE and Edwards-TL, after assuming the observations within the same participants over the 11 sessions were independent. First, the data were tested for multivariate normality of the residuals, and homogeneous variance-covariance of matrices across the groups (i.e., type of training modes) were checked using the chi-square quantile-quantile plot (for the multivariate normality assumption) and the Box's M test (for homogeneity of the variance-covariance matrices). The assumptions were all satisfied. Secondly, a multivariate analysis of variance (MANOVA) was performed with Bonferroni post-hoc analysis to determine if there were the same significant differences in the three types of training modes (contemporary, ballet, and rehearsal) when analysed by the two different session intensity methods (session-RPE and Edwards-TL). Thirdly, to determine the level of relationship between session-RPE and EdwardsTL, four simple linear regressions were performed (overall and by training mode: contemporary, ballet, and rehearsal) for Edwards-TL (dependent variable) and session-RPE (independent variable). All analyses were conducted using SPSS ver. 23 (IBM Corp., Armonk, NY, USA) and significance was set at $p \leq 0.05$.

\section{RESULTS}

A significant main effect was reported between the sessions $(F=13.39, p<0.001)$ and between-subject effects for session-
$\operatorname{RPE}\left(F_{2,93}=36.67 ; p<0.001\right)$ and Edwards-TL $\left(F_{2,93}=17.95\right.$; $p<0.001)$. For both session-RPE and Edwards-TL Bonferroni post hoc tests reported the contemporary sessions were significantly greater than the ballet and rehearsal sessions $(p<0.01)$, and there were no significant differences between the ballet and rehearsal sessions. For both session-RPE and Edwards-TL, the contemporary sessions had the greatest mean data, followed by ballet and finally rehearsal (Table 1 ).

Figure 1 shows the scatter plots of Edwards-TL and session-RPE, stratified by training mode. The result of the linear regression model, with Edwards-TL as the dependent variable and the session-RPE as the independent variable, indicates a significant large positive relationship $\left(r=0.72 ; F_{1,95}=101.1 ; p=0.001\right)$. The adjusted $r^{2}(0.513)$ and SEE $( \pm 53.96)$ indicate a poor predictive capability.

Analysis of the individual training modes indicated that rehearsals had the better relationship $(r=0.744$; $\left.F_{1,25}=29.69 ; p=0.001\right)$, though the adjusted $r^{2}(0.534)$ and SEE $( \pm 39.03)$ still indicate a poor predictive capability. The ballet $\left(r=0.462 ; F_{1,25}=6.507 ; p=0.018\right)$ and contemporary $\left(r=0.573 ; F_{1,25}=20.52 ; p=0.001\right)$ class sessions were both significant though the adjusted $r^{2}(0.181$ vs 0.312$)$, and the SEE $( \pm 57.78$ vs 60.14$)$ shows relatively poor predictive capabilities compared to rehearsal sessions.

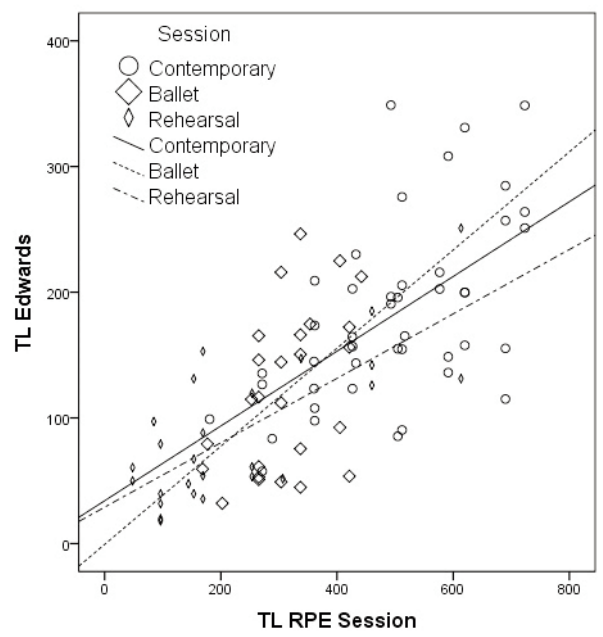

FIGURE 1. Scatter plot of Edwards-TL and session-RPE for each session type. < $\underline{\mathrm{AU}}$ : provide hi-res image $>$ 


\section{DISCUSSION}

This is the first study to investigate the construct validity of session-RPE as an indicator of ITL in vocational dance students using the correlation (convergent validity) between session-RPE and Edwards-LT as valid indicators of ITL. In this investigation, statistically significant positive correlations were found between session-RPE and Edwards-TL $(r=0.46-0.74)$. The magnitude of correlation between session-RPE method and Edwards-TL method were similar to those reported in previous investigations conducted in team sports such as soccer (1), 2010), ${ }^{27}$ and Australian football (Scott et al., 2013), < AU: give refs $>$ which all show moderate to very large relationships between session-RPE and HR-based methods for quantifying training loads.

The results of the present study indicate that the contemporary dance sessions recorded significantly higher scores for both session-RPE and Edwards-TL than ballet or rehearsal sessions and that there was no statistically significant difference between rehearsal and ballet sessions for either monitoring methods. This would suggest that students were working at higher intensity during contemporary dance classes and mirrors cardiorespiratory data previously reported (ballet class, $14.5 \pm 2.1 \mathrm{~mL} / \mathrm{kg} / \mathrm{min}^{28}$; contemporary dance class, $20.4 \pm 4.8 \mathrm{~mL} / \mathrm{kg} / \mathrm{min}^{29}$; and contemporary dance rehearsals, $10.17-17.19 \mathrm{~mL} / \mathrm{kg} / \mathrm{min}^{30}$ ).

Dance is a complex, diverse, non-steady-state, intermittent activity of moderate to high intensity and has notable differences between training, rehearsal, and performance intensities and durations. ${ }^{30-33}$ Impellizzeri et al. $(2004)^{25}$ claimed that the intermittent exercise nature of team sports (aerobic and anaerobic sources) could reduce the grade of correlation between session-RPE and Edwards-TL based methods. This would explain the range of observed relationships between session-RPE and Edwards-TL in the present study. This is further supported by research that reported an increase in participants RPE during intermittent protocols in comparison with a steady-state exercise session matched for total work, despite no difference in mean $\mathrm{VO}_{2}$ and $\mathrm{HR}$ between the two exercise protocols. ${ }^{34}$

Another finding in the current study is the variable magnitude of the correlations between different modes of training. A very large positive relationship was observed for rehearsal $(r=0.74)$, a large positive relationship was observed for contemporary $(r=0.573)$, and a moderate positive relationship for ballet $(r=0.462)$. The reason for these variations in correlation relationship is unclear. It could be speculated, however, that it is because session-RPE is derived from a subjective interpretation of exertion which combines a multitude of interrelated factors. For example, environmental factors such as temperature, music, feedback, and instruction ${ }^{17}$ could all alter perception of exercise intensity.

Moreover, a recent review article by Beck, Redding, and Wyon ${ }^{32}$ highlights the variability in energy demands of different dance genres, which poses challenges to drawing consensual conclusions across studies. The researchers go further to suggest that to report on the energy demands of dance more accurately, research should be dance genre specific, with detailed accounts of technical and stylistic elements of movement vocabulary and the inclusion of the reporting of work-to-rest ratios.

Understanding the training environment and the response that a multitude of external training factors may induce in an athlete/dancer is important when examining the effectiveness of session-RPE in monitoring ITL. Gaudino et al. ${ }^{35}$ investigated the relationship between internal and external load parameters in elite soccer and demonstrated that external factors significantly influenced load during training as assessed by session-RPE. They concluded that a multi-variant model more accurately assesses training load. Similarly, Minganti et al. ${ }^{16}$ demonstrated session-RPE is a valid means of measuring ITL for competitive divers but suggested a weighting factor (degree of diffculty) might be added to a calculation for session-RPE to more accurately reflect ITL when athletes practice sequences of different levels of technical difficulty. The findings of these two studies indicate that a combination of different external factors may better predict ITL than one individual parameter (session-RPE) alone. It is clear further research is needed to determine exactly what external training load factors most influence dancers' sessionRPE. Nevertheless, despite the possible influence of contributing external factors, literature supports the validity of session-RPE as an indicator of exercise intensity. ${ }^{17,36}$

It is reasonable to assume that the results of the present study may have been influenced by the small number of participant and the relatively short data collection period. Further research is required on a larger number of participants over longer periods of time to generate more conclusive results. However, it should be noted that the finding in this study align with previous studies validating session RPE against HR response with greater numbers of participants and larger data collections. . $^{12,16,17}$

\section{Recommendations and Conclusion}

In the present study, the magnitude of association between session-RPE and Edwards-TL is strong enough to provide confirmation that session-RPE can be an effective means of quantifying ITL in dance. The simplicity and versatility of the session-RPE method make it a valuable tool for dancers, teachers, and dance scientists if they wish to monitor the training process by quantifying the ITL as a single term. By quantifying ITL into a monitoring system of daily activity load scores, it can show patterns of weekly, monthly, and yearly training load. ${ }^{37,38}$ Furthermore, the factors such as the low cost and not having to rely on technical expertise or equipment make it a user-friendly and practical tool for monitoring ITL in dance. Understanding how these external training factors affect session-RPE would help dancers, teachers, and dance scientists to monitor training load more accurately. This notwithstanding, additional studies are strongly recommended to identify 
which external training factors are most influential on session-RPE in different training modes.

\section{REFERENCES}

1. Koutedakis Y. Burnout in dance: the physiological viewpoint. J Dance Med Sci. 2000;4(4):122-127.

2. Wyon M. Cardiorespiratory training for dancers. J Dance Med Sci. 2005;9(1):7-12.

3. Laws H. Fit to Dance 2-Report of the Second National Inquiry into Dancers' Health and Injury in the UK. London: Newgate Press; 2005.

4. Wyon M. Preparing to perform: periodization and dance. $J$ Dance Med Sci. 2010;14(2):67-72.

5. Bompa T, Haff G. Periodisation: Theory and Methodology of Training. Champaign, IL: Human Kinetics; 2009.

6. Koutedakis Y, Clarke F, Wyon M, et al. Muscular strength: applications for dancers. Med Probl Perform Artists. 2009; 24(4):157-165.

7. Rowbottom D. Periodization of training. In: Garret W, Kirkendall D, eds. Exercise and Sport Science. Philadelphia: Lippincott, Williams and Wilkins; 2000: pp499-512.

8. Barroso R, Cardoso R, do Carmo E, Tricoli V. Perceived exertion in coaches and young swimmers with different training experience. Int J Sports Physiol Perform. 2014;9(2):212-216. https:/ / doi.org/10.1123/ijspp.2012-0356.

9. Murgia C. Overuse, fatigue, and injury: neurological, psychological, physiological, and clinical aspects. J Dance Med Sci. 2013; 17(2):51-52.

10. Morton R, Fitz-Clarke J, Banister E. Modeling human performance in running. J Appl Physiol. 1990;69(3):1171-1177. https: / / doi.org/10.1152/jappl.1990.69.3.1171.

11. Banister E. Modeling elite athletic performance. In: Tanner R, Gore C, eds. Physiological Testing of Elite Athletes. Champaign, IL: Human Kinetics; 1991: pp403-424.

12. Foster C, Florhaug J, Franklin J, et al. A new approach to monitoring exercise training. J Strength Cond Res. 2001;15(1):109-115.

13. Foster C. Monitoring training in athletes with reference to overtraining syndrome. Med Sci Sports Exerc. 1998;30:1164-1168.

14. Sietsema K, Daly J, Wasserman K. Early dynamics of O2 uptake and heart rate as affected by exercise work rate. J Appl Physiol. 1989;67:2535-2541. https:/ / doi.org/10.1152/jappl.1989.67.6.2535.

15. Edwards S. The Heart Rate Monitor Book. Sacramento, CA: Fleet Feet Press; 1993.

16. Minganti C, Capranica L, Meeusen R, et al. The use of sessionRPE method for quantifying training load in diving. Int J Sports Physiol Perform. 2011;6(3):408-418. https://doi.org/10.1123/ ijspp.6.3.408.

17. Haddad M, Padulo J, Chamari K. The usefulness of session rating of perceived exertion for monitoring training load despite several influences on perceived exertion. Int J Sports Physiol Perform. 2014;9(5):882-883. https:/ /doi.org/10.1123/ijspp.2014-0010.

18. Lupo C, Capranica L, Tessitore A. The validity of the sessionRPE method for quantifying training load in water polo. Int $J$ Sports Physiol Perform. 2014;9(4):656-660. https://doi.org/10. 1123/ijspp.2013-0297.

19. Redding E, Wyon M, Shearman J, Doggart L. Validity of using heart rate as a predictor of oxygen consumption in dance. $J$ Dance Med Sci. 2004;8(3):69-72.

20. Lothian F, Farrally M. A comparison of methods for estimating oxygen uptake during intermittent exercise. J Sports Sci. 1995; 13:491-497. https:/ / doi.org/10.1080/02640419508732266.

21. Borg G. Psychological assessments of physical effort. Presented at: International Symposium on Psychological Assessment in Sport, 1978, Wingate Institute for Physical Education and Sport, Netanya, Israel.

22. Borg E, Kaijser L. A comparison between three rating scales for perceived exertion and two different work tests. Scand J Med Sci Sports. 2006;16(1):57-69. https://doi.org/10.1111/j.1600-0838. 2005.00448.x

23. Borresen J, Lambert M. The quantification of training load, the training response and the effect on performance. Sports Med. 2009;39(9):779-795. https://doi.org/10.2165/11317780000000000-00000.

24. Bannister W, Morton R, Fitz-Clarke J. Dose/response effects of exercise modeled from training: physical and biochemical measures. Ann Physiol Anthropol. 1992;11(3):345-356.

25. Impellizzeri F, Rampinini E, Coutts A, et al. Use of RPE-based training load in soccer. Med Sci Sports Exerc. 2004;36(6):10421047.

26. Wallace L, Slattery K, Coutts A. The ecological validity and application of the session-RPE method for quantifying training loads in swimming. J Strength Cond Res. 2009;23(1):33-38. https:/ / doi.org/10.1519/JSC.0b013e3181874512.

27. Manzi V, D'Ottavio S, Impellizzeri F, et al. Profile of weekly training load in elite male professional basketball players. $J$ Strength Cond Res. 2010;24(5):1399-1406. https://doi.org/10. 1519/JSC.0b013e3181d7552a.

28. Rodrigues-Krause J, Krause M, dos Santos Cunha G, et al. Ballet dancers cardiorespiratory, oxidative and muscle damage responses to classes and rehearsals. Eur J Sports Sci. 2014; 14(3):119-208. https://doi.org/10.1080/17461391.2013.777796.

29. Wyon M, Head A, Sharp C, Redding E. The cardiorespiratory responses to modern dance classes: differences between university, graduate, and professional classes. J Dance Med Sci. 2002; 6(2):41-45.

30. Wyon M, Abt G, Redding E, Head A, Sharp N. Oxygen uptake during of modern dance class, rehearsal and performance. $J$ Strength Cond Res. 2004;18(3):646-649. https:/ / doi.org/10.1519/ 13082.1.

31. Schantz P, Astrand P-O. Physiological characteristics of classical ballet. Med Sci Sports Exerc. 1984;16(5):472-476.

32. Beck S, Redding E, Wyon M. Methodological considerations for documenting the energy demand of dance activity: a review. Front Psychol. 2015;6. https: / / doi.org/10.3389/fpsyg.2015.00568.

33. Wyon M, Twitchett E, Angioi M, et al. Time motion and video analysis of classical ballet and contemporary dance performance. Int J Sports Med. 2011;32(11):851-855. https:/ / doi.org/10. 1055/s-0031-1279718.

34. Drust B, Reilly T, Cable N. Physiological responses to laboratory-based soccer-specific intermittent and continuous exercise. J Sports Sci. 2000;18(11):885-892. https://doi.org/10.1080/ 026404100750017814.

35. Gaudino P, Iaia F, Strudwick A, et al. Factors influencing perception of effort (session rating of perceived exertion) during elite soccer training. Int J Sports Physiol Perform. 2015;10(7):860-864. https: / / doi.org/10.1123/ijspp.2014-0518.

36. Turner AN, Buttigieg C, Marshall G, Net al. Ecological validity of the session rating of perceived exertion for quantifying internal training load in fencing. Int J Sports Physiol Perform. 2017;12(1):124-128. https:/ / doi.org/10.1123/ijspp.2016-0062.

37. Wyon M. Towards a new training methodology. In: Brown D, Vos M, eds. Ballet, How and Why?: On the Role of Classical Ballet in Dance Education. Arnhem, Neth.: ArtEZ Press; 2014: pp111118.

38. Menaspà MJ, Menaspà $\mathrm{P}$, Clark SA, Fanchini M. Validity of the online athlete management system to assess training load. Int $J$ Sports Physiol Perform. 2018:13(6):750-754. https://doi.org/10. 1123 /ijspp.2017-0379.

\section{$<$ AU: pls check refs for missing DOI numbers $>$}

Received 17-Nov-2017, accepted 8-June-2018

Published online 1-Mar-2019

https: / / doi.org/10.21091/mppa.2019.1002 